Research article

Open Access

\title{
Nonsteroidal anti-inflammatory drugs and breast cancer risk in the National Institutes of Health-AARP Diet and Health Study
}

\author{
Gretchen L Gierach ${ }^{1}, 2$, James V Lacey Jr ${ }^{1}$, Arthur Schatzkin ${ }^{3}$, Michael F Leitzmann ${ }^{3}$, \\ Douglas Richesson ${ }^{1}$, Albert R Hollenbeck ${ }^{4}$ and Louise A Brinton ${ }^{1}$
}

\begin{abstract}
${ }^{1}$ Hormonal and Reproductive Epidemiology Branch, Division of Cancer Epidemiology and Genetics, National Cancer Institute, National Institutes of Health, 6120 Executive Blvd, Suite 550, Rockville, MD 20852-7234, USA

${ }^{2}$ Cancer Prevention Fellowship Program, Office of Preventive Oncology, National Cancer Institute, National Institutes of Health, 6120 Executive Blvd, Suite T-41, Bethesda, MD 20892-7361, USA

${ }^{3}$ Nutritional Epidemiology Branch, Division of Cancer Epidemiology and Genetics, National Cancer Institute, National Institutes of Health, 6120

Executive Blvd, Suite 320, Rockville, MD 20852-7232, USA

${ }^{4}$ Organizational and Tracking Research Department, AARP, 601 E Street NW, Washington, DC 20049, USA
\end{abstract}

Corresponding author: Gretchen L Gierach, gierachg@mail.nih.gov

Received: 17 Dec 2007 Revisions requested: 14 Feb 2008 Revisions received: 18 Mar 2008 Accepted: 30 Apr 2008 Published: 30 Apr 2008

Breast Cancer Research 2008, 10:R38 (doi:10.1186/bcr2089)

This article is online at: http://breast-cancer-research.com/content/10/2/R38

(C) 2008 Gierach et al.; licensee BioMed Central Ltd.

This is an open access article distributed under the terms of the Creative Commons Attribution License (http://creativecommons.org/licenses/by/2.0), which permits unrestricted use, distribution, and reproduction in any medium, provided the original work is properly cited.

\begin{abstract}
Introduction By inhibiting cyclooxygenase-2, nonsteroidal antiinflammatory drugs (NSAIDs) decrease aromatase activity and might reduce breast cancer risk by suppressing estrogen synthesis. Epidemiologic evidence for a protective role of NSAIDs in breast cancer, however, is equivocal.

Methods We tested NSAID use for its association with breast cancer incidence in the National Institutes of Health-AARP Diet and Health Study, where 127,383 female AARP (formerly known as the American Association of Retired Persons) members with no history of cancer, aged 51 to 72 years, completed a mailed questionnaire (1996 to 1997). We estimated relative risks of breast cancer for NSAID exposures using multivariate Cox proportional hazards regression models. The state cancer registry and mortality index linkage identified 4,501 primary incident breast cancers through 31 December 2003 , including 1,439 estrogen receptor (ER)-positive cancers and 280 ER-negative cancers.
\end{abstract}

Results Proportional hazards models revealed no statistically significant association between overall NSAIDs and total breast cancer. As cyclooxygenase inhibition by aspirin (but not other NSAIDs) is irreversible, we tested associations by NSAID type. Although we observed no significant differences in risk for daily use (versus nonuse) of aspirin (relative risk $=0.93,95 \%$ confidence interval $=0.85$ to 1.01 ) or nonaspirin NSAIDS (relative risk $=0.96,95 \%$ confidence interval $=0.87$ to 1.05 ), risk of ER-positive breast cancer was significantly reduced with daily aspirin use (relative risk $=0.84,95 \%$ confidence interval $=$ 0.71 to 0.98 ) - a relationship not observed for nonaspirin NSAIDS. Neither aspirin nor nonaspirin NSAIDs were associated with risk of ER-negative breast cancer.

Conclusion Breast cancer risk was not significantly associated with NSAID use, but daily aspirin use was associated with a modest reduction in ER-positive breast cancer. Our results provide support for further evaluating relationships by NSAID type and breast cancer subtype.

\section{Introduction}

Numerous epidemiologic studies have examined the association between nonsteroidal anti-inflammatory drugs (NSAIDs) and breast cancer, with equivocal results. Most, but not all, case-control studies have found relative risk reductions from $20 \%$ to $40 \%$ [1-3] (reviewed in [4-7]). Results from prospective studies have been less consistent, with eight reports finding no association [8-15], two studies observing an increased risk [16,17], one study reporting a U-shaped association [18], and six studies demonstrating a reduced risk [19-24]. In the Women's Health Study randomized clinical trial, alternate-day use of low-dose aspirin for an average of 10 years did not reduce the incidence of breast cancer [25]. Given the potential public health impact of NSAIDs as chemopreventive agents for breast cancer, an increased understanding of the relationship between NSAIDs and breast cancer is needed.

$\mathrm{BMI}=$ body mass index; $\mathrm{Cl}=$ confidence interval; $\mathrm{COX}=$ cyclooxygenase; $\mathrm{ER}=$ estrogen receptor; $\mathrm{HR}=$ hormone receptor; $\mathrm{HT}=$ hormone therapy; $\mathrm{NIH}=$ National Institutes of Health; NSAID = nonsteroidal anti-inflammatory drug; RR = relative risk. 
A protective role of NSAIDs in breast carcinogenesis is biologically plausible. In vitro and animal studies have consistently shown that NSAIDs inhibit cyclooxygenase (COX)-1 and COX-2, which cyclize and oxygenate arachidonic acid, eventually producing prostaglandins [26]. While inhibiting COX-1 reduces both platelet aggregation and gastric cytoprotection [27], inactivating COX-2 may interrupt the carcinogenic process in the breast via multiple pathways - including inhibition of angiogenesis, promotion of apoptosis, and suppression of estrogen synthesis via decreased aromatase activity [28].

Since aspirin and nonaspirin NSAIDs have different biologic effects (aspirin permanently inactivates COX-2 [29]), separate evaluation is needed with respect to breast cancer risk. Interpretation of prior epidemiologic studies is complicated by incomplete attention to NSAID type [5]. Furthermore, the studies that have examined the effect of NSAIDs on breast cancer risk by tumor characteristics, such as hormone receptor (HR) status $[3,13,16-18,23,24,30,31]$ and stage at diagnosis $[21,30,32]$, also present conflicting results. The relatively few studies that have tested for effect modification by participant characteristics (for example, body mass index, menopausal hormone therapy use, or smoking status) have produced inconclusive findings $[13,17,18,20,22,24,25,32,33]$. The large cohort of women participating in the prospective National Institutes of Health (NIH)-AARP Diet and Health Study allowed us to evaluate these questions.

\section{Materials and methods Study population}

The NIH-AARP Diet and Health Study design and methodology have been described in detail [34]. Briefly, the NIH-AARP Study is a prospective cohort study of diet, health-related behaviors and cancer.

The study was initiated in 1995 and 1996 when a questionnaire was mailed to 3.5 million members of the AARP (formerly known as the American Association of Retired Persons) aged 50 to 71 years, who resided in one of six states (California, Florida, Pennsylvania, New Jersey, North Carolina, and Louisiana) or two metropolitan areas (Atlanta, Georgia and Detroit, Michigan) that have large AARP membership, large minority populations, and high-quality cancer registries. The questionnaire captured diet history, demographic characteristics, reproductive history, menopausal status, and family history of cancer. Of the 617,119 men and women (17.6\%) who returned the questionnaire, 567,169 satisfactorily completed it.

In 1996 and 1997 a second questionnaire was sent to collect additional information on family history of cancer, anthropometry, and use of menopausal hormone therapy $(\mathrm{HT})$ and other medications, including NSAIDs. A total of 337,074 men and women completed this questionnaire.
After excluding individuals who died or moved out of the cancer registry ascertainment area before their second questionnaire was received and scanned $(n=2,166)$, excluding proxy respondents to the baseline $(n=6,959)$ or second $(n=$ $3,424)$ questionnaires, and excluding 188,117 men, the study population included 136,408 potentially eligible women

The study was approved by the Special Studies Institutional Review Board of the US National Cancer Institute.

\section{Assessment of nonsteroidal anti-inflammatory drug exposure and covariates}

The second questionnaire asked whether aspirin products (generic aspirin, Bayer, Bufferin, Anacin, Ecotrin, or Excedrin) and nonaspirin NSAIDs (generic ibuprofen, Advil, Nuprin, Motrin, Naprosyn, and so on) had been used in the past 12 months. As acetaminophen is an analgesic with weak antiinflammatory activity [35], participants were instructed not to include 'Tylenol, acetaminophen, or any other pain relievers' in nonaspirin NSAIDs. Aspirin or nonaspirin NSAID users were asked to indicate their frequency of usual use: fewer than 2 times per month, 2 to 3 times per month, 1 to 2 times per week, 3 to 4 times per week, 5 to 6 times per week, 1 time per day, or 2 or more times per day - which we collapsed to categories of nonuse, $<1 /$ week, 1 to $6 /$ week, and $\geq 1 /$ day. The dose, duration, and indication for use were not collected. In the current report, 'any NSAID' combines the use of aspirin and nonaspirin NSAIDs.

While the demographic characteristics, lifestyle, and reproductive history were largely obtained through the first questionnaire, the second questionnaire ascertained a selfreported history of hypertension, mammogram screening, and vigorous physical activity. Menopausal hormone therapy use and history of breast cancer in a first-degree relative were derived from information collected on both the first and second questionnaires.

\section{Cohort follow-up}

Cohort members were followed annually for mailing address changes and vital status. Address changes were identified through linkage to the US Postal Service's National Change of Address database, through US Postal Service updates received with undeliverable mail, through use of other address change update services, and through participants' notifications. Vital status was updated through linkage to the Social Security Administration Death Master File and was verified by the National Death Index.

\section{Ascertainment of breast cancer}

Incident in situ breast cancers and invasive breast cancers were initially identified through probabilistic linkage to eight state cancer registries using first and last name, address, sex, date of birth, and Social Security Number from the baseline questionnaire. The cancer registry ascertainment area was 
recently expanded to include three additional states (Texas, Arizona, and Nevada) to capture cancer occurring among participants who moved to those states during follow-up. Each registry has been certified by the North American Association of Central Cancer Registries for meeting the highest standards of data quality.

Breast cancer estrogen receptor (ER) status was coded as described in the American Joint Committee on Cancer's Collaborative Staging Site-Specific Factors Manual, with a threshold of $>10 \mathrm{fmol}$ cytosol protein per milligram for a positive tumor; however, the ER status was not reported by the Florida, Michigan, and Pennsylvania cancer registries. Histology was defined using the International Classification of Diseases for Oncology codes, third edition [36]. A previous validation study in this cohort estimated that registry linkage validly identified approximately $90 \%$ of all incident cancers [37]. The date of death for fatal cancers was identified through linkage to the National Death Index.

\section{Analytic sample}

Because information regarding NSAID use was not collected on the baseline questionnaire, we limited analyses to the 136,408 women who completed the second questionnaire. We excluded 9,022 women who reported a personal cancer history other than nonmelanoma skin cancer on either questionnaire (including 942 breast cancers), 1,259 women who were missing information on NSAID use (including 52 breast cancers), and three women with no follow-up. Thus, 126,124 women were included in the present analyses.

Through 31 December 2003, 4,501 women developed breast cancer (781 in situ, 3,703 invasive, and 17 missing stage). Among the 2,282 invasive breast cancer cases from states that collected the ER status, 1,439 were coded as ER-positive and 280 as ER-negative (the ER status was unavailable for the remaining 563 invasive cases). There were no substantial risk factor differences between women from states where ER information was available and states where it was unavailable. The majority of invasive breast cancers were ductal carcinomas $(n=2,409)$, followed by lobular $(n=400)$, mixed $(n=$ $310)$, and other $(n=584)$ histologic types.

\section{Statistical analysis}

Cox proportional hazards models were used to estimate hazard ratios and 95\% confidence intervals (Cls) for breast cancer associated with NSAID use; age was the time scale [38], and ties were handled by enumeration [39]. Follow-up began at the age at which the second questionnaire was received and scanned, and continued through the earliest of the following dates: participant diagnosed with breast cancer, moved out of her registry catchment area, died from any cause, or 31 December 2003. To test the proportional hazards assumption, we generated time-dependent covariates by including interactions of each predictor with the natural log of age (the time metric); probability values for all time-dependent covariates were $>0.05$, consistent with hazards that are proportional.

Multivariate models were used to control for age at entry (years), race/ethnicity (white versus other/unknown), age at first birth (nulliparous, $<20$ years, 20 to 24 years, 25 to 29 years, $\geq 30$ years, or unknown), HT use (never used, estrogen only, estrogen-progestin, or other/unknown), number of breast biopsies $(0,1,2, \geq 3$, or unknown), alcoholic drinks per day $(0,<1,1$ to $3, \geq 3$, or unknown), history of hypertension (no, yes, or unknown), and family history of breast cancer in a firstdegree relative (no, yes, or unknown). Models examining frequency of aspirin use and breast cancer risk also included terms for frequency of nonaspirin NSAID use and vice versa. Tests for linear trends across the NSAID exposure categories were calculated by treating these categorical variables as ordinal variables.

In subsequent models, we adjusted for calendar time and several additional factors, including ages at menarche and menopause, mammogram in the past 3 years, self-reported heart disease, self-rated health quality, physical activity, and body mass index (BMI). The results were essentially the same and are not shown here.

We used a likelihood ratio test, comparing models with and without the interaction terms, to separately examine effect modification by age at entry ( $<57$ years, 57 to 60 years, 61 to 64 years, 65 to 68 years, or $\geq 69$ years), HT use (never, estrogen only, estrogen-progestin, or other/unknown), BMI (<25 $\mathrm{kg} / \mathrm{m}^{2}, 25$ to $29 \mathrm{~kg} / \mathrm{m}^{2}, \geq 30 \mathrm{~kg} / \mathrm{m}^{2}$, or unknown), and smoking status (never, former, current, or unknown). In addition, we examined whether the relationship between NSAIDs and breast cancer incidence differed by ER status (positive or negative), by stage at diagnosis (in situ or invasive disease), and by histologic type (ductal, lobular, or mixed).

Probability values $<0.05$ were considered statistically significant. All tests of statistical significance were two-tailed. Analyses were performed using SAS software release 9.1.3 (SAS Institute Inc., Cary, NC, USA).

\section{Results}

Among the 126,124 mostly white, postmenopausal women in this report, $65.6 \%$ reported ever-use of aspirin in the past 12 months. Among all women, 31.8\% took aspirin less than once per week, $16.1 \%$ used aspirin one to six times per week, and $17.7 \%$ were daily aspirin users. Slightly fewer women reported ever-use of nonaspirin NSAIDs: $31.5 \%$ took nonaspirin NSAIDs less than once per week, 15.2\% used nonaspirin NSAIDs one to six times per week, and $13.3 \%$ were daily nonaspirin NSAID users. Nearly $85 \%$ of women in our study reported any NSAID use within the past 12 months. 
In general, aspirin and nonaspirin NSAID users shared similar characteristics (Tables 1 and 2). Compared with women who did not use aspirin or nonaspirin NSAIDs in the past 12 months, women who had used aspirin or nonaspirin NSAIDs on a daily basis were more likely to be white, and to reportedly drink alcohol, smoke, use HT, have fair/poor overall health, and to report a history of hypertension. Daily aspirin users were more likely to be older and to report a history of stroke and heart disease. Daily nonaspirin NSAID users were more likely to be younger, parous, and obese $\left(\mathrm{BMI} \geq 30 \mathrm{~kg} / \mathrm{m}^{2}\right)$, and to have a history of breast biopsy.

The 126,124 women accrued 836,863 person-years during an average follow-up of 3.43 years for cases (range, 1 day to 7.13 years) and of 6.75 years for noncases (range, 1 day to 7.17 years). The mean (standard deviation) ages for entry and exit were $63.1(5.2)$ years and $66.5(5.6)$ years for cases and were 62.6 (5.4) years and 69.3 (5.4) years for noncases, respectively. Breast cancer risk factors in this population were generally consistent with established associations with age, $\mathrm{BMI}$, ages at menarche, first birth and menopause, parity, estrogen-progestin therapy use, number of breast biopsies, and family history of breast cancer.

Because NSAID exposure was collected on the second questionnaire, and the response rate for this questionnaire was $\sim 60 \%$, we also examined the association between breast cancer risk factors captured on the first questionnaire and breast cancer risk among the entire cohort. The associations between risk factors and breast cancer in the entire cohort were comparable with those observed in the subcohort of respondents to the second questionnaire (data not shown).

\section{Nonsteroidal anti-inflammatory drug use and breast cancer}

In multivariate proportional hazards models, there was no statistically significant association between ever-use of NSAIDs in the past 12 months and total breast cancer (Table 3). Compared with nonusers, the relative risks (RRs) of breast cancer associated with ever-use of aspirin only, with nonaspirin NSAIDs only, or with both were $0.97(95 \% \mathrm{Cl}=0.88$ to 1.07$)$, $1.01(95 \% \mathrm{Cl}=0.92$ to 1.12$)$, and $0.95(95 \% \mathrm{Cl}=0.87$ to 1.04 ), respectively. We tested associations by frequency of NSAID type. The risk of total breast cancer was slightly reduced with daily use (versus nonuse) of aspirin ( $R R=0.93$, $95 \% \mathrm{Cl}=0.85$ to 1.01 ), albeit statistically nonsignificantly (for trend $P=0.08$ ). Compared with nonuse, daily use of nonaspirin NSAIDs was not associated with breast cancer risk ( $R R=$ $0.96,95 \% \mathrm{Cl}=0.87$ to 1.05$)$. The association between NSAID use and breast cancer risk was not modified by age, HT use, BMl, or smoking (data not shown).

Several differences emerged in analyses stratified by ER status and stage (Tables 4 to 6 ). The RR of ER-positive breast cancer was reduced with daily aspirin use (RR $=0.84,95 \%$
$\mathrm{Cl}=0.71$ to 0.98$)$, and no association was observed with daily use of nonaspirin NSAIDs ( $R R=0.98,95 \% \mathrm{Cl}=0.83$ to 1.16 ) (Table 4). In contrast, ER-negative breast cancer was not associated with daily aspirin or nonaspirin NSAID use. The inverse relationships with aspirin use were weak for invasive breast cancer but were stronger for in situ disease (Table 5), and these differences were not entirely explained by mammographic screening: in analyses restricted to women who reported having two or more mammograms in the past three years, the risks associated with daily aspirin use for invasive and in situ breast cancers were $0.95(95 \% \mathrm{Cl}=0.85$ to 1.06) and $0.75(95 \% \mathrm{Cl}=0.59$ to 0.95$)$, respectively (Table 6$)$. There was little variation in risks for nonaspirin NSAIDs according to ER status and stage. Risk relationships for aspirin and nonaspirin NSAIDs did not vary by histologic subtype for invasive cancers (data not shown).

\section{Discussion}

In the present large prospective study, we found that NSAID use was unrelated to risk of total breast cancer. Daily aspirin, but not nonaspirin NSAID, use was associated with a modest $16 \%$ reduction in ER-positive breast cancer. We did not observe an association between NSAID use and ER-negative breast cancer.

Our null findings for total breast cancer are consistent with those reported by eight prospective studies [8-15] and one randomized clinical trial [25] that examined aspirin and/or nonaspirin NSAID use. Our results differ, however, from those from seven prospective studies [17,19-24] and 12 case-control studies [1-3,30-33,40-44], most of which have reported reduced risks of breast cancer associated with NSAID use. The reasons for these differing results are unclear, but associations may be limited to certain subtypes of breast cancer. Differences in NSAID exposure assessment across studies may also account for inconsistent results.

The frequency of aspirin and nonaspirin use in the NIH-AARP Diet and Health Study is consistent with that reported in other US cohorts of women [21,45], and the proportion of women in this study using any NSAID is only slightly higher than that reported among females ages 45 to $75+$ years in the third National Health and Nutrition Examination Survey $-71.5 \%$ to $80.4 \%$ of whom reported any nonprescription analgesic in the past month [46]. Our study lacked information, however, on the duration, dose, indication, or prescription NSAID use. Because we only measured recent NSAID use in the year prior to the 1996 to 1997 questionnaire and followed women through 2003, the null findings we observed for NSAIDs may differ from what might be observed with long-term cumulative exposure. Indeed, several cohort studies have shown that long-term NSAID use is required for an observed chemopreventive effect on breast cancer [22,23]. Furthermore, if there were individuals in our referent category who were past users 
Distribution of select risk factors across categories of aspirin use among 126,124 women, National Institutes of Health-AARP Study

\begin{tabular}{|c|c|c|c|c|c|c|c|c|}
\hline \multirow[b]{3}{*}{ Characteristic } & \multicolumn{8}{|c|}{ Frequency of aspirin use in past 12 months } \\
\hline & \multicolumn{2}{|l|}{ None } & \multicolumn{2}{|l|}{$<$ weekly } & \multicolumn{2}{|c|}{$1-6$ times/week } & \multicolumn{2}{|l|}{$1+/$ day } \\
\hline & $n$ & $\%{ }^{a}$ & $n$ & $\%^{\mathrm{a}}$ & $n$ & $\%^{\mathrm{a}}$ & $n$ & $\%{ }^{a}$ \\
\hline \multicolumn{9}{|l|}{ Age at second questionnaire } \\
\hline$<57$ years & 8,178 & 19.0 & 8,629 & 21.7 & 4,029 & 20.1 & 2,936 & 13.3 \\
\hline $57-60$ years & 8,317 & 19.4 & 8,211 & 20.6 & 4,022 & 20.1 & 3,566 & 16.2 \\
\hline $61-64$ years & 9,750 & 22.7 & 9,025 & 22.7 & 4,644 & 23.2 & 5,132 & 23.2 \\
\hline $65-68$ years & 11,085 & 25.8 & 9,459 & 23.8 & 4,881 & 24.3 & 6,607 & 29.9 \\
\hline $69+$ years & 5,630 & 13.1 & 4,457 & 11.2 & 2,481 & 12.4 & 3,839 & 17.4 \\
\hline \multicolumn{9}{|l|}{ Race/ethnicity } \\
\hline Caucasian/non-Hispanic white & 38,339 & 89.2 & 36,340 & 91.4 & 18,561 & 92.5 & 20,381 & 92.3 \\
\hline Other/unknown & 4,621 & 10.8 & 3,441 & 8.6 & 1,496 & 7.5 & 1,699 & 7.7 \\
\hline \multicolumn{9}{|l|}{ Education } \\
\hline$<$ High school/high school grad & 13,537 & 32.5 & 10,675 & 27.5 & 5,769 & 29.6 & 6,857 & 32.0 \\
\hline Post-high school+ & 28,171 & 67.5 & 28,110 & 72.5 & 13,696 & 70.4 & 14,554 & 68.0 \\
\hline \multicolumn{9}{|l|}{ Body mass index at baseline } \\
\hline$<25 \mathrm{~kg} / \mathrm{m}^{2}$ & 18,461 & 44.7 & 18,855 & 48.9 & 9,040 & 46.6 & 8,718 & 40.8 \\
\hline $25-30 \mathrm{~kg} / \mathrm{m}^{2}$ & 13,144 & 31.8 & 12,331 & 32.0 & 6,407 & 33.0 & 7,290 & 34.2 \\
\hline $30+\mathrm{kg} / \mathrm{m}^{2}$ & 9,719 & 23.5 & 7,342 & 19.1 & 3,965 & 20.4 & 5,335 & 25.0 \\
\hline \multicolumn{9}{|l|}{ Smoking } \\
\hline Never & 19,321 & 46.3 & 18,123 & 47.0 & 8,996 & 46.4 & 8,984 & 42.1 \\
\hline Former & 16,758 & 40.2 & 15,314 & 39.7 & 7,735 & 39.9 & 9,184 & 43.0 \\
\hline Current & 5,630 & 13.5 & 5,137 & 13.3 & 2,656 & 13.7 & 3,193 & 14.9 \\
\hline \multicolumn{9}{|l|}{ Alcoholic drinks } \\
\hline 0 drinks per day & 14,515 & 33.8 & 9,136 & 23.0 & 4,784 & 23.9 & 7,009 & 31.7 \\
\hline$<1$ drink per day & 23,322 & 54.3 & 24,771 & 62.3 & 12,299 & 61.3 & 12,111 & 54.9 \\
\hline $1-3$ drinks per day & 4,037 & 9.4 & 4,727 & 11.9 & 2,382 & 11.9 & 2,297 & 10.4 \\
\hline $3+$ drinks per day & 1,086 & 2.5 & 1,147 & 2.9 & 592 & 3.0 & 663 & 3.0 \\
\hline \multicolumn{9}{|l|}{ Self-reported general health } \\
\hline Excellent/very good/good & 36,189 & 85.6 & 36,638 & 93.3 & 17,869 & 90.4 & 17,868 & 82.4 \\
\hline Fair/poor & 6,094 & 14.4 & 2,639 & 6.7 & 1,891 & 9.6 & 3,814 & 17.6 \\
\hline \multicolumn{9}{|l|}{ Stroke } \\
\hline No & 42,293 & 98.4 & 39,557 & 99.4 & 19,844 & 98.9 & 21,112 & 95.6 \\
\hline Yes & 667 & 1.6 & 224 & 0.6 & 213 & 1.1 & 968 & 4.4 \\
\hline \multicolumn{9}{|l|}{ Heart disease } \\
\hline No & 39,820 & 92.7 & 38,292 & 96.3 & 18,924 & 94.4 & 17,561 & 79.5 \\
\hline Yes & 3,140 & 7.3 & 1,489 & 3.7 & 1,133 & 5.6 & 4,519 & 20.5 \\
\hline \multicolumn{9}{|l|}{ High blood pressure } \\
\hline No & 24,240 & 60.2 & 25,179 & 67.7 & 11,484 & 61.5 & 9,502 & 46.1 \\
\hline Yes & 16,001 & 39.8 & 11,997 & 32.3 & 7,192 & 38.5 & 11,117 & 53.9 \\
\hline \multicolumn{9}{|l|}{ Mammogram in past 3 years } \\
\hline Once or less & 13,226 & 31.0 & 12,161 & 30.8 & 6,043 & 30.4 & 6,496 & 29.8 \\
\hline Yes, more than once & 29,408 & 69.0 & 27,298 & 69.2 & 13,827 & 69.6 & 15,327 & 70.2 \\
\hline
\end{tabular}


Breast Cancer Research Vol 10 No 2 Gierach et al.

Table 1 (Continued)

Distribution of select risk factors across categories of aspirin use among 126,124 women, National Institutes of Health-AARP Study Age at menarche

\begin{tabular}{|c|c|c|c|c|c|c|c|c|}
\hline$<13$ years & 21,222 & 49.8 & 18,850 & 47.8 & 9,679 & 48.7 & 11,036 & 50.4 \\
\hline $13-14$ years & 17,452 & 41.0 & 17,101 & 43.4 & 8,407 & 42.3 & 8,859 & 40.5 \\
\hline $15+$ years & 3,909 & 9.2 & 3,492 & 8.9 & 1,792 & 9.0 & 1,981 & 9.1 \\
\hline \multicolumn{9}{|l|}{ Parity } \\
\hline Nulliparous & 6,253 & 14.8 & 5,996 & 15.3 & 2,808 & 14.2 & 3,089 & 14.3 \\
\hline One & 4,459 & 10.6 & 4,056 & 10.4 & 2,009 & 10.2 & 2,172 & 10.0 \\
\hline Two & 11,052 & 26.2 & 10,488 & 26.8 & 5,290 & 26.8 & 5,406 & 25.0 \\
\hline Three or more & 20,431 & 48.4 & 18,578 & 47.5 & 9,608 & 48.7 & 10,987 & 50.7 \\
\hline \multicolumn{9}{|l|}{ Age at first live birth } \\
\hline Nulliparous & 6,253 & 14.8 & 5,996 & 15.3 & 2,808 & 14.2 & 3,089 & 14.2 \\
\hline$<20$ years & 7,385 & 17.4 & 6,016 & 15.3 & 3,319 & 16.8 & 3,895 & 17.9 \\
\hline $20-24$ years & 18,472 & 43.6 & 17,112 & 43.6 & 8,861 & 44.9 & 9,800 & 45.1 \\
\hline $25-29$ years & 7,636 & 18.0 & 7,582 & 19.3 & 3,632 & 18.4 & 3,739 & 17.2 \\
\hline $30+$ years & 2,575 & 6.1 & 2,499 & 6.4 & 1,129 & 5.7 & 1,191 & 5.5 \\
\hline \multicolumn{9}{|l|}{ Age at menopause } \\
\hline Premenopausal & 1,462 & 3.4 & 1,738 & 4.4 & 742 & 3.7 & 445 & 2.0 \\
\hline$<45$ years & 2,834 & 6.6 & 2,429 & 6.1 & 1,266 & 6.3 & 1,593 & 7.2 \\
\hline $45-49$ years & 6,543 & 15.2 & 6,316 & 15.9 & 2,997 & 14.9 & 3,358 & 15.2 \\
\hline $50-54$ years & 11,362 & 26.4 & 11,870 & 29.8 & 5,415 & 27.0 & 5,732 & 26.0 \\
\hline $55+$ years & 2,650 & 6.2 & 2,555 & 6.4 & 1,218 & 6.1 & 1,284 & 5.8 \\
\hline Surgical menopause & 16,770 & 39.0 & 13,577 & 34.1 & 7,754 & 38.7 & 8,975 & 40.6 \\
\hline Postmenopausal, age unknown & 1,339 & 3.1 & 1,296 & 3.3 & 665 & 3.3 & 693 & 3.1 \\
\hline \multicolumn{9}{|l|}{ Hormone therapy formulation } \\
\hline Never used & 17,328 & 42.4 & 15,676 & 41.2 & 7,201 & 37.6 & 8,371 & 39.8 \\
\hline Estrogen therapy only & 11,901 & 29.1 & 10,157 & 26.7 & 5,844 & 30.5 & 6,724 & 32.0 \\
\hline Estrogen-progestin therapy & 11,681 & 28.6 & 12,196 & 32.1 & 6,100 & 31.9 & 5,945 & 28.3 \\
\hline \multicolumn{9}{|c|}{ Moderate/vigorous physical activity during past 10 years } \\
\hline Never & 1,801 & 4.3 & 1,033 & 2.6 & 545 & 2.8 & 878 & 4.0 \\
\hline Rarely & 4,911 & 11.6 & 4,012 & 10.2 & 1,986 & 10.1 & 2,539 & 11.7 \\
\hline$<1$ hour/week & 4,473 & 10.6 & 4,102 & 10.5 & 2,107 & 10.7 & 2,347 & 10.8 \\
\hline 1-3 hours/week & 10,495 & 24.9 & 10,085 & 25.7 & 5,240 & 26.6 & 5,457 & 25.1 \\
\hline 4-7 hours/week & 10,379 & 24.6 & 10,438 & 26.6 & 5,311 & 26.9 & 5,371 & 24.7 \\
\hline$>7$ hours/week & 10,100 & 24.0 & 9,548 & 24.3 & 4,546 & 23.0 & 5,122 & 23.6 \\
\hline \multicolumn{9}{|l|}{ Number breast biopsies } \\
\hline None & 32,240 & 75.8 & 30,183 & 76.6 & 14,985 & 75.4 & 16,551 & 75.8 \\
\hline One & 6,819 & 16.0 & 6,175 & 15.7 & 3,217 & 16.2 & 3,428 & 15.7 \\
\hline Two & 1,904 & 4.5 & 1,666 & 4.2 & 926 & 4.7 & 997 & 4.6 \\
\hline Three or more & 1,581 & 3.7 & 1,360 & 3.5 & 737 & 3.7 & 863 & 4.0 \\
\hline \multicolumn{9}{|c|}{ Family history of breast cancer in first degree relative (male or female) } \\
\hline No & 29,536 & 83.5 & 27,782 & 83.8 & 13,814 & 83.6 & 14,930 & 83.4 \\
\hline Yes & 5,838 & 16.5 & 5,356 & 16.2 & 2,708 & 16.4 & 2,978 & 16.6 \\
\hline
\end{tabular}

aMissing values were excluded from percentage calculations. 
Distribution of select risk factors across categories of nonaspirin nonsteroidal anti-inflammatory drug (NSAID) use among 126,124 women, National Institutes of Health-AARP Study

\begin{tabular}{|c|c|c|c|c|c|c|c|c|}
\hline \multirow[b]{3}{*}{ Characteristic } & \multicolumn{8}{|c|}{ Frequency of nonaspirin NSAID use in past 12 months } \\
\hline & \multicolumn{2}{|l|}{ None } & \multicolumn{2}{|l|}{$<$ weekly } & \multicolumn{2}{|c|}{$1-6$ times/week } & \multicolumn{2}{|l|}{$1+/$ day } \\
\hline & $n$ & $\%^{\mathrm{a}}$ & $n$ & $\%{ }^{\mathrm{a}}$ & $n$ & $\%{ }^{\mathrm{a}}$ & $n$ & $\%^{\mathrm{a}}$ \\
\hline \multicolumn{9}{|l|}{ Age at second questionnaire } \\
\hline$<57$ years & 7,112 & 14.3 & 9,185 & 23.4 & 4,563 & 24.0 & 2,955 & 17.9 \\
\hline $57-60$ years & 8,605 & 17.3 & 8,288 & 21.1 & 4,171 & 21.9 & 3,052 & 18.4 \\
\hline $61-64$ years & 11,482 & 23.0 & 8,854 & 22.5 & 4,278 & 22.5 & 3,876 & 23.4 \\
\hline $65-68$ years & 14,666 & 29.4 & 8,841 & 22.5 & 4,039 & 21.2 & 4,399 & 26.6 \\
\hline $69+$ years & 7,962 & 16.0 & 4,136 & 10.5 & 1,962 & 10.3 & 2,263 & 13.7 \\
\hline \multicolumn{9}{|l|}{ Race/ethnicity } \\
\hline Caucasian/non-Hispanic white & 44,909 & 90.1 & 35,940 & 91.4 & 17,537 & 92.2 & 15,103 & 91.3 \\
\hline Other/unknown & 4,918 & 9.9 & 3,364 & 8.6 & 1,476 & 7.8 & 1,442 & 8.7 \\
\hline \multicolumn{9}{|l|}{ Education } \\
\hline$<$ High school/high school grad & 16,007 & 33.1 & 10,402 & 27.1 & 5,595 & 30.3 & 4,734 & 29.5 \\
\hline Post-high school+ & 32,364 & 66.9 & 27,959 & 72.9 & 12,851 & 69.7 & 11,312 & 70.5 \\
\hline \multicolumn{9}{|l|}{ Body mass index at baseline } \\
\hline$<25 \mathrm{~kg} / \mathrm{m}^{2}$ & 23,279 & 48.6 & 18,527 & 48.6 & 7,800 & 42.4 & 5,418 & 33.9 \\
\hline $25-30 \mathrm{~kg} / \mathrm{m}^{2}$ & 15,189 & 31.7 & 12,423 & 32.6 & 6,194 & 33.7 & 5,296 & 33.2 \\
\hline $30+\mathrm{kg} / \mathrm{m}^{2}$ & 9,464 & 19.7 & 7,193 & 18.9 & 4,413 & 24.0 & 5,254 & 32.9 \\
\hline \multicolumn{9}{|l|}{ Smoking } \\
\hline Never & 22,702 & 47.0 & 17,354 & 45.5 & 8,254 & 44.8 & 7,062 & 44.0 \\
\hline Former & 18,554 & 38.4 & 15,751 & 41.3 & 7,708 & 41.9 & 6,942 & 43.2 \\
\hline Current & 7,025 & 14.6 & 5,023 & 13.2 & 2,453 & 13.3 & 2,050 & 12.8 \\
\hline \multicolumn{9}{|l|}{ Alcoholic drinks } \\
\hline 0 drinks per day & 16,225 & 32.6 & 9,230 & 23.5 & 4,728 & 24.9 & 5,121 & 31.0 \\
\hline$<1$ drink per day & 27,087 & 54.4 & 24,388 & 62.0 & 11,660 & 61.3 & 9,312 & 56.3 \\
\hline $1-3$ drinks per day & 5,108 & 10.3 & 4,570 & 11.6 & 2,153 & 11.3 & 1,616 & 9.8 \\
\hline $3+$ drinks per day & 1,407 & 2.8 & 1,116 & 2.8 & 472 & 2.5 & 496 & 3.0 \\
\hline \multicolumn{9}{|l|}{ Self-reported general health } \\
\hline Excellent/very good/good & 42,891 & 87.5 & 35,824 & 92.4 & 16,655 & 88.8 & 13,073 & 80.5 \\
\hline Fair/poor & 6,142 & 12.5 & 2,966 & 7.6 & 2,106 & 11.2 & 3,176 & 19.5 \\
\hline \multicolumn{9}{|l|}{ Stroke } \\
\hline No & 48,762 & 97.9 & 38,882 & 98.9 & 18,760 & 98.7 & 16,223 & 98.1 \\
\hline Yes & 1,065 & 2.1 & 422 & 1.1 & 253 & 1.3 & 322 & 1.9 \\
\hline \multicolumn{9}{|l|}{ Heart disease } \\
\hline No & 44,964 & 90.2 & 36,916 & 93.9 & 17,658 & 92.9 & 14,936 & 90.3 \\
\hline Yes & 4,863 & 9.8 & 2,388 & 6.1 & 1,355 & 7.1 & 1,609 & 9.7 \\
\hline \multicolumn{9}{|l|}{ High blood pressure } \\
\hline No & 27,666 & 59.6 & 23,960 & 65.1 & 10,656 & 60.1 & 8,036 & 51.7 \\
\hline Yes & 18,783 & 40.4 & 12,847 & 34.9 & 7,080 & 39.9 & 7,508 & 48.3 \\
\hline \multicolumn{9}{|l|}{ Mammogram in past 3 years } \\
\hline Once or less & 17,403 & 35.3 & 10,768 & 27.6 & 5,095 & 27.0 & 4,509 & 27.5 \\
\hline Yes, more than once & 31,949 & 64.7 & 28,229 & 72.4 & 13,749 & 73.0 & 11,904 & 72.5 \\
\hline
\end{tabular}


Table 2 (Continued)

Distribution of select risk factors across categories of nonaspirin nonsteroidal anti-inflammatory drug (NSAID) use among 126,124 women, National Institutes of Health-AARP Study

\begin{tabular}{|c|c|c|c|c|c|c|c|c|}
\hline \multicolumn{9}{|l|}{ Age at menarche } \\
\hline$<13$ years & 23,714 & 48.1 & 18,717 & 48.0 & 9,436 & 50.0 & 8,816 & 53.7 \\
\hline $13-14$ years & 21,018 & 42.6 & 16,749 & 43.0 & 7,752 & 41.1 & 6,269 & 38.2 \\
\hline $15+$ years & 4,604 & 9.3 & 3,516 & 9.0 & 1,685 & 8.9 & 1,336 & 8.1 \\
\hline \multicolumn{9}{|l|}{ Parity } \\
\hline Nulliparous & 8,010 & 16.4 & 5,540 & 14.3 & 2,375 & 12.7 & 2,212 & 13.6 \\
\hline One & 5,330 & 10.9 & 4,022 & 10.4 & 1,794 & 9.6 & 1,546 & 9.5 \\
\hline Two & 12,441 & 25.5 & 10,535 & 27.2 & 5,125 & 27.3 & 4,095 & 25.2 \\
\hline Three or more & 23,059 & 47.2 & 18,566 & 48.0 & 9,445 & 50.4 & 8,418 & 51.7 \\
\hline \multicolumn{9}{|l|}{ Age at first live birth } \\
\hline Nulliparous & 8,010 & 16.4 & 5,540 & 14.3 & 2,375 & 12.6 & 2,212 & 13.6 \\
\hline$<20$ years & 7,690 & 15.7 & 6,056 & 15.6 & 3,559 & 19.0 & 3,229 & 19.8 \\
\hline $20-24$ years & 21,059 & 43.0 & 17,194 & 44.4 & 8,585 & 45.7 & 7,350 & 45.1 \\
\hline $25-29$ years & 9,058 & 18.5 & 7,520 & 19.4 & 3,310 & 17.6 & 2,676 & 16.4 \\
\hline $30+$ years & 3,153 & 6.4 & 2,439 & 6.3 & 948 & 5.0 & 844 & 5.2 \\
\hline \multicolumn{9}{|l|}{ Age at menopause } \\
\hline Premenopausal & 1,145 & 2.3 & 1,864 & 4.7 & 886 & 4.7 & 487 & 2.9 \\
\hline$<45$ years & 3,654 & 7.3 & 2,377 & 6.0 & 1,063 & 5.6 & 995 & 6.0 \\
\hline $45-49$ years & 8,216 & 16.5 & 5,941 & 15.1 & 2,732 & 14.4 & 2,288 & 13.8 \\
\hline $50-54$ years & 14,355 & 28.8 & 11,383 & 29.0 & 4,705 & 24.7 & 3,895 & 23.5 \\
\hline $55+$ years & 3,209 & 6.4 & 2,519 & 6.4 & 1,019 & 5.4 & 945 & 5.7 \\
\hline Surgical menopause & 17,713 & 35.5 & 13,938 & 35.5 & 7,962 & 41.9 & 7,430 & 44.9 \\
\hline Postmenopausal, age unknown & 1,535 & 3.1 & 1,282 & 3.3 & 646 & 3.4 & 505 & 3.1 \\
\hline \multicolumn{9}{|l|}{ Hormone therapy formulation } \\
\hline Never used & 23,083 & 48.6 & 14,199 & 37.8 & 5,961 & 32.9 & 5,179 & 32.8 \\
\hline Estrogen therapy only & 12,664 & 26.7 & 10,499 & 27.9 & 5,910 & 32.7 & 5,522 & 35.0 \\
\hline Estrogen-progestin therapy & 11,762 & 24.8 & 12,876 & 34.3 & 6,224 & 34.4 & 5,072 & 32.2 \\
\hline \multicolumn{9}{|c|}{ Moderate/vigorous physical activity during past 10 years } \\
\hline Never & 1,922 & 3.9 & 1,004 & 2.6 & 551 & 2.9 & 749 & 4.6 \\
\hline Rarely & 5,434 & 11.1 & 3,953 & 10.2 & 1,993 & 10.6 & 2,032 & 12.5 \\
\hline$<1$ hour/week & 5,034 & 10.3 & 4,196 & 10.8 & 1,980 & 10.6 & 1,815 & 11.2 \\
\hline 1-3 hours/week & 12,127 & 24.8 & 10,252 & 26.4 & 4,815 & 25.7 & 4,091 & 25.2 \\
\hline 4-7 hours/week & 12,378 & 25.3 & 10,169 & 26.2 & 4,981 & 26.6 & 3,924 & 24.1 \\
\hline$>7$ hours/week & 12,017 & 24.6 & 9,234 & 23.8 & 4,397 & 23.5 & 3,642 & 22.4 \\
\hline \multicolumn{9}{|l|}{ Number breast biopsies } \\
\hline None & 37,903 & 76.9 & 29,547 & 75.9 & 14,113 & 74.9 & 12,243 & 74.7 \\
\hline One & 7,549 & 15.3 & 6,236 & 16.0 & 3,114 & 16.5 & 2,727 & 16.6 \\
\hline Two & 2,042 & 4.1 & 1,779 & 4.6 & 884 & 4.7 & 768 & 4.7 \\
\hline Three or more & 1,793 & 3.6 & 1,373 & 3.5 & 743 & 3.9 & 652 & 4.0 \\
\hline \multicolumn{9}{|c|}{ Family history of breast cancer in first degree relative (male or female) } \\
\hline No & 33,976 & 83.6 & 27,478 & 83.7 & 13,197 & 83.8 & 11,295 & 82.8 \\
\hline Yes & 6,643 & 16.4 & 5,346 & 16.3 & 2,554 & 16.2 & 2,347 & 17.2 \\
\hline
\end{tabular}

aMissing values were excluded from percentage calculations. 


\begin{tabular}{|c|c|c|c|c|c|}
\hline & $\begin{array}{l}\text { Number of } \\
\text { cancers }\end{array}$ & Person-years & Relative riska & $\begin{array}{l}95 \% \text { confidence } \\
\text { interval }\end{array}$ & $\begin{array}{l}P \text { value } \\
\text { for trend }\end{array}$ \\
\hline \multicolumn{6}{|l|}{ NSAID use } \\
\hline Never & 667 & 124,265 & 1.00 (referent) & & $\mathrm{n} / \mathrm{a}$ \\
\hline Aspirin only & 1,085 & 203,145 & 0.97 & 0.88 to 1.07 & \\
\hline Non-aspirin NSAID only & 874 & 157,017 & 1.01 & 0.92 to 1.12 & \\
\hline Both & 1,781 & 334,792 & 0.95 & 0.87 to 1.04 & \\
\hline \multicolumn{6}{|l|}{ Aspirin use ${ }^{b}$} \\
\hline Never & 1,556 & 284,342 & 1.00 (referent) & & 0.08 \\
\hline$<1 /$ week & 1,405 & 265,676 & 0.95 & 0.89 to 1.03 & \\
\hline $1-6 /$ week & 716 & 133,331 & 0.95 & 0.87 to 1.04 & \\
\hline $1+/$ day & 774 & 145,317 & 0.93 & 0.85 to 1.01 & \\
\hline \multicolumn{6}{|l|}{ Non-aspirin NSAID use ${ }^{b}$} \\
\hline Never & 1,764 & 329,944 & 1.00 (referent) & & 0.58 \\
\hline$<1 /$ week & 1,415 & 262,000 & 1.00 & 0.93 to 1.07 & \\
\hline $1-6 /$ week & 693 & 126,373 & 1.02 & 0.93 to 1.11 & \\
\hline $1+/$ day & 585 & 109,098 & 0.96 & 0.87 to 1.05 & \\
\hline
\end{tabular}

aAjusted for age (continuous), race, age at first birth, hormone therapy use, number of breast biopsies, alcohol intake, history of hypertension, and family history of breast cancer in first-degree relative.

bThese models also include terms for frequency of use of opposite NSAID type.

or prescription NSAID users, results could be biased toward the null.

In addition, we were unable to separate low-dose from regulardose aspirin use; results from the Women's Health Study randomized trial [25], a study conducted using an insurance database [2], and laboratory evidence [27] suggest that doses higher than the $80 \mathrm{mg}$ aspirin per day typically recommended for cardiovascular therapy may be required to permanently inactivate COX-2. If low-dose aspirin users were included in our exposed group, thereby diluting any effect associated with regular-dose aspirin use, the results would be biased toward the null. Although we did not collect information on indication for use, it is likely that some daily users were taking aspirin for heart disease prevention given that daily users more frequently reported history of heart disease. To address the concern that women using NSAIDS for cardioprotection may be under closer medical supervision and/or may be more health conscious, we additionally controlled for self-reported heart disease, self-rated health quality, and mammographic screening - and the results remained unchanged.

Despite these limitations, the NIH-AARP Diet and Health Study is the one of largest cohorts to date to have evaluated the association between NSAID type and breast cancer risk by tumor characteristics, including ER status. The reduced risk of
ER-positive breast cancer we observed with daily aspirin use is consistent with the mechanism of action of aspirin (but not nonaspirin NSAIDs), which permanently inactivates COX-2 [29], potentially reducing breast cancer risk via multiple pathways, including suppression of estrogen synthesis by decreased aromatase activity [28]. In addition, our results are in agreement with findings from the Long Island Breast Cancer Prevention Project, a population-based case-control study that reported in 2004 an inverse association with aspirin that was significantly greater for HR-positive breast cancer than for HR-negative breast cancer [30]. Since then, two case-control studies [3,31] and three prospective cohort studies $[16,17,24]$ have separately investigated the association for aspirin, with three of the five studies suggesting some protection against HR-positive breast cancer with aspirin use $[3,16,31]$.

A US hospital-based study - the Case-Control Surveillance Study - reported a $26 \%$ nonsignificant reduction $(95 \% \mathrm{Cl}=$ 0.44 to 1.26 ) in HR-positive breast cancer associated with regular (versus <4 times/week) aspirin use [31]. More recently, a Canadian population-based case-control study found a $31 \%$ reduction $(95 \% \mathrm{Cl}=0.56$ to 0.86 ) in ER-positive/PR-positive breast cancer associated with daily (versus $<$ daily) aspirin use [3]. The California Teachers Study followed 114,460 women aged 22 to 85 years for 6 years and found 
Table 4

Association between nonsteroidal anti-inflammatory drug (NSAID) use and breast cancer by estrogen receptor status, National Institutes of Health-AARP Study

\begin{tabular}{|c|c|c|c|c|c|c|c|c|c|c|}
\hline \multirow[b]{2}{*}{ NSAID use } & \multicolumn{5}{|c|}{ Estrogen receptor-positive } & \multicolumn{5}{|c|}{ Estrogen receptor-negative } \\
\hline & $\begin{array}{l}\text { Number of } \\
\text { cancers }\end{array}$ & $\begin{array}{l}\text { Person- } \\
\text { years }\end{array}$ & Relative risk ${ }^{\mathrm{a}}$ & $\begin{array}{l}95 \% \text { confidence } \\
\text { interval }\end{array}$ & $\begin{array}{l}P \text { value } \\
\text { for trend }\end{array}$ & $\begin{array}{l}\text { Number } \\
\text { of cancers }\end{array}$ & $\begin{array}{l}\text { Person- } \\
\text { years }\end{array}$ & Relative risk ${ }^{a}$ & $\begin{array}{l}95 \% \text { confidence } \\
\text { interval }\end{array}$ & $\begin{array}{l}P \text { value } \\
\text { for trend }\end{array}$ \\
\hline \multicolumn{11}{|l|}{ Aspirin use ${ }^{b}$} \\
\hline Never & 493 & 280,706 & 1.00 (referent) & & 0.06 & 88 & 279,308 & 1.00 (referent) & & 0.54 \\
\hline$<1 /$ week & 464 & 262,491 & 0.98 & 0.86 to 1.11 & & 93 & 261,137 & 1.09 & 0.81 to 1.47 & \\
\hline $1-6 /$ week & 243 & 131,724 & 1.00 & 0.86 to 1.17 & & 42 & 131,022 & 1.01 & 0.70 to 1.47 & \\
\hline $1+/$ day & 223 & 143,476 & 0.84 & 0.71 to 0.98 & & 52 & 142,852 & 1.14 & 0.81 to 1.62 & \\
\hline \multicolumn{11}{|l|}{$\begin{array}{l}\text { Nonaspirin } \\
\text { NSAID use }\end{array}$} \\
\hline Never & 541 & 325,816 & 1.00 (referent) & & 0.86 & 108 & 324,250 & 1.00 (referent) & & 0.64 \\
\hline$<1 /$ week & 466 & 258,767 & 1.04 & 0.92 to 1.18 & & 96 & 257,460 & 1.08 & 0.81 to 1.43 & \\
\hline $1-6 /$ week & 230 & 124,804 & 1.07 & 0.92 to 1.26 & & 36 & 124,115 & 0.85 & 0.58 to 1.25 & \\
\hline $1+/$ day & 186 & 107,763 & 0.98 & 0.83 to 1.16 & & 36 & 107,241 & 0.97 & 0.66 to 1.42 & \\
\hline
\end{tabular}

The threshold for a positive estrogen receptor was $\geq 10 \mathrm{fmol}$ receptor/mg total protein. aAdjusted for age (continuous), race, age at first birth, hormone therapy use, number of breast biopsies, alcohol intake, history of hypertension, and family history of breast cancer in first-degree relative. bThese models also include terms for frequency of use of opposite NSAID type.

that long-term daily (versus <once/week) aspirin use was associated with a statistically nonsignificant decreased risk of ER-positive/PR-positive breast cancer $(\mathrm{RR}=0.80,95 \% \mathrm{Cl}=$ 0.62 to 1.03 ) [16]. In contrast, the Multiethnic Cohort Study and the Danish Diet, Cancer and Health Cohort Study found no protective effect of aspirin use for HR-positive or HR-negative breast cancer $[17,24]$. Finally, the Women's Health Study randomized clinical trial did not observe any significant patterns of risk by HR status with low-dose aspirin use [25]. Therefore, while our findings, along with several others, suggest at least some reduction in ER-positive breast cancer associated with aspirin use, the evidence is not conclusive and additional prospective studies with detailed exposure data on

Table 5

Association between nonsteroidal anti-inflammatory drug (NSAID) use and breast cancer according to stage, National Institutes of Health-AARP Study

\begin{tabular}{|c|c|c|c|c|c|c|c|c|c|c|}
\hline \multirow[b]{2}{*}{ NSAID use } & \multicolumn{5}{|c|}{ In situ breast cancer } & \multicolumn{5}{|c|}{ Invasive breast cancer } \\
\hline & $\begin{array}{l}\text { Number of } \\
\text { cancers }\end{array}$ & $\begin{array}{l}\text { Person- } \\
\text { years }\end{array}$ & Relative risk ${ }^{\mathrm{a}}$ & $\begin{array}{l}95 \% \text { confidence } \\
\text { interval }\end{array}$ & $\begin{array}{l}P \text { value } \\
\text { for trend }\end{array}$ & $\begin{array}{l}\text { Number of } \\
\text { cancers }\end{array}$ & $\begin{array}{l}\text { Person- } \\
\text { years }\end{array}$ & Relative risk ${ }^{\mathrm{a}}$ & $\begin{array}{l}95 \% \text { confidence } \\
\text { interval }\end{array}$ & $\begin{array}{l}P \text { value } \\
\text { for trend }\end{array}$ \\
\hline \multicolumn{11}{|l|}{ Aspirin use $\mathrm{e}^{\mathrm{b}}$} \\
\hline Never & 298 & 280,077 & 1.00 (referent) & & 0.02 & 1,254 & 283,247 & 1.00 (referent) & & 0.37 \\
\hline$<1 /$ week & 233 & 261,641 & 0.80 & 0.67 to 0.96 & & 1,166 & 264,821 & 0.99 & 0.91 to 1.07 & \\
\hline $1-6 /$ week & 124 & 131,305 & 0.85 & 0.69 to 1.05 & & 589 & 132,899 & 0.98 & 0.88 to 1.08 & \\
\hline 1+/day & 122 & 143,137 & 0.78 & 0.63 to 0.96 & & 648 & 144,854 & 0.96 & 0.87 to 1.06 & \\
\hline \multicolumn{11}{|l|}{$\begin{array}{l}\text { Non-aspirin } \\
\text { NSAID use }\end{array}$} \\
\hline Never & 292 & 324,890 & 1.00 (referent) & & 0.45 & 1,463 & 328,884 & 1.00 (referent) & & 0.88 \\
\hline$<1 /$ week & 269 & 258,097 & 1.14 & 0.96 to 1.35 & & 1,140 & 261,013 & 0.97 & 0.90 to 1.05 & \\
\hline 1-6/week & 122 & 124,433 & 1.06 & 0.85 to 1.31 & & 569 & 125,934 & 1.01 & 0.92 to 1.12 & \\
\hline $1+/$ day & 91 & 107,466 & 0.87 & 0.69 to 1.10 & & 494 & 108,757 & 0.98 & 0.89 to 1.09 & \\
\hline
\end{tabular}

aAdjusted for age (continuous), race, age at first birth, hormone therapy use, number of breast biopsies, alcohol intake, history of hypertension, and family history of breast cancer in first-degree relative.

bThese models also include terms for frequency of use of opposite NSAID type. 
Association between nonsteroidal anti-inflammatory drug (NSAID) use and breast cancer according to stage among screened women, National Institutes of Health-AARP Study

\begin{tabular}{|c|c|c|c|c|c|c|c|c|c|c|}
\hline \multirow[b]{2}{*}{ NSAID use } & \multicolumn{5}{|c|}{ In situ breast cancer } & \multicolumn{5}{|c|}{ Invasive breast cancer } \\
\hline & $\begin{array}{l}\text { Number of } \\
\text { cancers }\end{array}$ & $\begin{array}{l}\text { Person- } \\
\text { years }\end{array}$ & Relative risk ${ }^{a}$ & $\begin{array}{l}95 \% \text { confidence } \\
\text { interval }\end{array}$ & $\begin{array}{l}P \text { value } \\
\text { for trend }\end{array}$ & $\begin{array}{l}\text { Number of } \\
\text { cancers }\end{array}$ & $\begin{array}{l}\text { Person- } \\
\text { years }\end{array}$ & Relative risk ${ }^{a}$ & $\begin{array}{l}95 \% \text { confidence } \\
\text { interval }\end{array}$ & $\begin{array}{l}P \text { value } \\
\text { for trend }\end{array}$ \\
\hline \multicolumn{11}{|l|}{ Aspirin use ${ }^{b}$} \\
\hline Never & 241 & 192,125 & 1.00 (referent) & & 0.02 & 916 & 194,396 & 1.00 (referent) & & 0.15 \\
\hline$<1 /$ week & 180 & 179,747 & 0.77 & 0.63 to 0.93 & & 847 & 181,986 & 0.98 & 0.89 to 1.07 & \\
\hline $1-6 /$ week & 97 & 90,680 & 0.82 & 0.65 to 1.05 & & 402 & 91,715 & 0.90 & 0.80 to 1.01 & \\
\hline $1+/$ day & 96 & 99,793 & 0.75 & 0.59 to 0.95 & & 476 & 101,064 & 0.95 & 0.85 to 1.06 & \\
\hline \multicolumn{11}{|l|}{$\begin{array}{l}\text { Non-aspirin } \\
\text { NSAID use }\end{array}$} \\
\hline Never & 218 & 209,044 & 1.00 (referent) & & 0.50 & 982 & 211,655 & 1.00 (referent) & & 0.62 \\
\hline$<1 /$ week & 222 & 185,624 & 1.16 & 0.95 to 1.40 & & 852 & 187,741 & 0.98 & 0.89 to 1.07 & \\
\hline $1-6 /$ week & 97 & 90,035 & 1.03 & 0.81 to 1.31 & & 428 & 91,161 & 1.03 & 0.92 to 1.16 & \\
\hline $1+/$ day & 76 & 77,462 & 0.89 & 0.69 to 1.16 & & 384 & 78,439 & 1.02 & 0.91 to 1.15 & \\
\hline
\end{tabular}

This analysis was restricted to women who reported $\geq 2$ mammograms in the past 3 years. aAdjusted for age (continuous), race, age at first birth, hormone therapy use, number of breast biopsies, alcohol intake, history of hypertension, and family history of breast cancer in first-degree relative. bThese models also include terms for frequency of use of opposite NSAID type.

the dose, frequency, duration, and indication are needed.

We found stronger inverse associations with in situ breast cancer than invasive breast cancer. Among the few prior studies that have reported results separately for in situ breast cancer and invasive breast cancer, two case-control studies reported decreased risks of both in situ and invasive breast cancers associated with aspirin [30] and with any NSAID use [32], but the findings for in situ breast cancer were not statistically significant in either study. The lowa Women's Health cohort study reported a reduction in breast cancer risk with increased frequency of aspirin, but not with nonaspirin NSAIDs, for both in situ and invasive disease [21]. Both in situ and invasive breast tumors express COX-2 [47], suggesting that upregulation of COX-2 may be an early event in carcinogenesis. Higher rates of COX-2 expression in in situ compared with invasive tumors have led investigators to suggest that the potential therapeutic impact of COX-2 inhibition may be more relevant for in situ breast cancer than invasive breast cancer (reviewed in [48]). The inverse association for in situ breast cancer associated with aspirin use in the present study may therefore be biologically plausible and warrants further investigation.

We observed no variation in risk with $\mathrm{HT}$, smoking status, or $\mathrm{BMI}$, and our findings are generally consistent with previous investigations. For the most part, tests for interactions between HT and NSAID use have been statistically nonsignificant across case-control studies [30,33] and cohort studies $[13,17,22,24,25]$; however, two cohort studies have suggested the protective effect of NSAIDs may be attenuated among HT users $[18,20]$. In line with data suggesting that proinflammatory tobacco carcinogens may alter the effectiveness of chemopreventive agents [49], the Women's Health Study found that low-dose aspirin use was protective among former smokers but was associated with an increased risk among never smokers (for interaction $P=0.09$ ) [25]. We, along with a Canadian population-based case-control study, however, did not observe effect modification by smoking [3]. Consistent with our findings, two case-control studies $[32,33$ and seven prospective studies $[13,17,18,20,22,24,25]$ have reported no significant interactions between BMI and NSAID use with respect to breast cancer risk.

\section{Conclusion}

In summary, our results do not support an important influence of NSAIDs on total breast cancer risk. Daily aspirin use, however, appeared to offer some protection for ER-positive breast cancer in this population. In addition, our findings suggest that the associations for aspirin use may vary by tumor stage. Our results provide support for further evaluating relationships in prospective studies with well-defined measures of NSAID use by NSAID type, by breast cancer stage, and by ER status.

\section{Competing interests}

The authors declare that they have no competing interests.

\section{Authors' contributions}

$A S, A R H$, and MFL participated in the acquisition of data. GLG, LAB, JVL Jr, AS, and MFL were involved with the study concept and design. GLG, JVL Jr, MFL, DR, and LAB contrib- 
uted to the statistical analyses. GLG, JVL Jr, and LAB participated in manuscript preparation. All authors participated in the interpretation of results and critical revision of the manuscript for important intellectual content. All authors read and approved the final manuscript.

\section{Acknowledgements}

The authors are indebted to the participants in the NIH-AARP Diet and Health Study for their outstanding cooperation. The authors thank Traci Mouw, former study coordinator of the NIH-AARP Diet and Health Study, for research assistance. This research was supported in part by the Intramural Research Program of the $\mathrm{NIH}$, National Cancer Institute.

Cancer incidence data from the Atlanta metropolitan area were collected by the Georgia Center for Cancer Statistics, Department of Epidemiology, Rollins School of Public Health, Emory University. Cancer incidence data from California were collected by the California Department of Health Services, Cancer Surveillance Section. Cancer incidence data from the Detroit metropolitan area were collected by the Michigan Cancer Surveillance Program, Community Health Administration, state of Michigan. The Florida cancer incidence data used in this report were collected by the Florida Cancer Data System under contract to the Department of Health. The views expressed herein are solely those of the authors and do not necessarily reflect those of the contractor or Department of Health. Cancer incidence data from Louisiana were collected by the Louisiana Tumor Registry, Louisiana State University Medical Center, New Orleans. Cancer incidence data from New Jersey were collected by the New Jersey State Cancer Registry, Cancer Epidemiology Services, New Jersey State Department of Health and Senior Services. Cancer incidence data from North Carolina were collected by the North Carolina Central Cancer Registry. Cancer incidence data from Pennsylvania were supplied by the Division of Health Statistics and Research, Pennsylvania Department of Health, Harrisburg, Pennsylvania. The Pennsylvania Department of Health specifically disclaims responsibility for any analyses, interpretations or conclusions.

\section{References}

1. Harris RE, Beebe-Donk J, Alshafie GA: Reduction in the risk of human breast cancer by selective cyclooxygenase-2 (COX-2) inhibitors. BMC Cancer 2006, 6:27.

2. Rahme E, Ghosn J, Dasgupta K, Rajan R, Hudson M: Association between frequent use of nonsteroidal anti-inflammatory drugs and breast cancer. BMC Cancer 2005, 5:159.

3. Kirsh VA, Kreiger N, Cotterchio M, Sloan M, Theis B: Nonsteroidal antiinflammatory drug use and breast cancer risk: subgroup findings. Am J Epidemiol 2007, 166:709-716.

4. Khuder SA, Mutgi AB: Breast cancer and NSAID use: a metaanalysis. Br J Cancer 2001, 84:1188-1192.

5. Harris RE, Beebe-Donk J, Doss H, Burr Doss D: Aspirin, ibuprofen, and other non-steroidal anti-inflammatory drugs in cancer prevention: a critical review of non-selective COX-2 blockade (review). Oncol Rep 2005, 13:559-583.

6. Bosetti C, Gallus S, La Vecchia C: Aspirin and cancer risk: an updated quantitative review to 2005. Cancer Causes Control 2006, 17:871-888

7. Mangiapane S, Blettner M, Schlattmann P: Aspirin use and breast cancer risk: a meta-analysis and meta-regression of observational studies from 2001 to 2005. Pharmacoepidemiol Drug Saf 2008, 17:115-124.

8. Paganini-Hill A, Chao A, Ross RK, Henderson BE: Aspirin use and chronic diseases: a cohort study of the elderly. BMJ 1989 , 299:1247-1250.

9. Egan KM, Stampfer MJ, Giovannucci E, Rosner BA, Colditz GA: Prospective study of regular aspirin use and the risk of breast cancer. J Natl Cancer Inst 1996, 88:988-993.

10. Friis S, Sorensen HT, McLaughlin JK, Johnsen SP, Blot WJ, Olsen $\mathrm{JH}$ : A population-based cohort study of the risk of colorectal and other cancers among users of low-dose aspirin. $\mathrm{Br} J$ Cancer 2003, 88:684-688.

11. Sorensen HT, Friis S, Norgard B, Mellemkjaer L, Blot WJ, McLaughlin JK, Ekbom A, Baron JA: Risk of cancer in a large cohort of nonaspirin NSAID users: a population-based study. Br J Cancer 2003, 88:1687-1692.

12. García Rodríguez LA, González-Pérez A: Risk of breast cancer among users of aspirin and other anti-inflammatory drugs. $\mathrm{Br}$ $J$ Cancer 2004, 91:525-529.

13. Jacobs EJ, Thun MJ, Connell CJ, Rodriguez C, Henley SJ, Feigelson HS, Patel AV, Flanders WD, Calle EE: Aspirin and other nonsteroidal anti-inflammatory drugs and breast cancer incidence in a large U.S. cohort. Cancer Epidemiol Biomarkers Prev 2005 , 14:261-264.

14. Jacobs EJ, Thun MJ, Bain EB, Rodriguez C, Henley SJ, Calle EE: A large cohort study of long-term daily use of adult-strength aspirin and cancer incidence. J Natl Cancer Inst 2007, 99:608-615

15. Vogel U, Christensen J, Nexo BA, Wallin H, Friis S, Tjonneland A: Peroxisome profilerator-activated receptorgamma2 Pro12Ala, interaction with alcohol intake and NSAID use, in relation to risk of breast cancer in a prospective study of Danes. Carcinogenesis 2007, 28:427-434.

16. Marshall SF, Bernstein L, Anton-Culver $H$, Deapen D, Horn-Ross $\mathrm{PL}$, Mohrenweiser H, Peel D, Pinder R, Purdie DM, Reynolds $P$, Stram D, West D, Wright WE, Ziogas A, Ross RK: Nonsteroidal anti-inflammatory drug use and breast cancer risk by stage and hormone receptor status. J Natl Cancer Inst 2005, 97:805-812

17. Friis $\mathrm{S}$, Thomassen $\mathrm{L}$, Sorensen $\mathrm{HT}$, Tjonneland $\mathrm{A}$, Overvad $\mathrm{K}$, Cronin-Fenton DP, Vogel U, McLaughlin JK, Blot WJ, Olsen JH: Nonsteroidal anti-inflammatory drug use and breast cancer risk: a Danish cohort study. Eur J Cancer Prev 2008, 17:88-96.

18. Ready A, Velicer CM, McTiernan A, White E: NSAID use and breast cancer risk in the VITAL cohort. Breast Cancer Res Treat 2007. DOI 10.1007/s10549-007-9665-x

19. Schreinemachers DM, Everson RB: Aspirin use and lung, colon, and breast cancer incidence in a prospective study. Epidemiology 1994, 5:138-146.

20. Harris RE, Kasbari S, Farrar WB: Prospective study of nonsteroidal anti-inflammatory drugs and breast cancer. Oncol Rep 1999, 6:71-73.

21. Johnson TW, Anderson KE, Lazovich D, Folsom AR: Association of aspirin and nonsteroidal anti-inflammatory drug use with breast cancer. Cancer Epidemiol Biomarkers Prev 2002, 11:1586-1591.

22. Harris RE, Chlebowski RT, Jackson RD, Frid DJ, Ascenseo JL, Anderson G, Loar A, Rodabough RJ, White E, McTiernan A, Women's Health Initiative: Breast cancer and nonsteroidal antiinflammatory drugs: prospective results from the Women's Health Initiative. Cancer Res 2003, 63:6096-6101.

23. Gallicchio L, Visvanathan K, Burke A, Hoffman SC, Helzlsouer KJ: Nonsteroidal anti-inflammatory drugs and the risk of developing breast cancer in a population-based prospective cohort study in Washington County, MD. Int J Cancer 2007, 121:211-215.

24. Gill JK, Maskarinec G, Wilkens LR, Pike MC, Henderson BE, Kolonel LN: Nonsteroidal antiinflammatory drugs and breast cancer risk: the multiethnic cohort. Am J Epidemiol 2007 166:1150-1158

25. Cook NR, Lee IM, Gaziano JM, Gordon D, Ridker PM, Manson JE, Hennekens $\mathrm{CH}$, Buring JE: Low-dose aspirin in the primary prevention of cancer: the Women's Health Study: a randomized controlled trial. JAMA 2005, 294:47-55.

26. Thun MJ, Henley SJ, Patrono C: Nonsteroidal anti-inflammatory drugs as anticancer agents: mechanistic, pharmacologic, and clinical issues. J Natl Cancer Inst 2002, 94:252-266.

27. Patrono C, Coller B, FitzGerald GA, Hirsh J, Roth G: Plateletactive drugs: the relationships among dose, effectiveness, and side effects: the Seventh ACCP Conference on Antithrombotic and Thrombolytic Therapy. Chest 2004, 126:234S-264S.

28. Singh-Ranger G, Salhab M, Mokbel K: The role of cyclooxygenase-2 in breast cancer: review. Breast Cancer Res Treat 2007. DOI 10.1007/s10549-007-9641-5

29. Kadar D: Anti-inflammatory analgesics. In Principles of Medical Pharmacology Edited by: Kalant H, Roschlau WHE. New York: Oxford University Press; 1998:410-430. 
30. Terry MB, Gammon MD, Zhang FF, Tawfik H, Teitelbaum SL, Britton JA, Subbaramaiah K, Dannenberg AJ, Neugut Al: association of frequency and duration of aspirin use and hormone receptor status with breast cancer risk. JAMA 2004, 291:2433-2440.

31. Zhang Y, Coogan PF, Palmer JR, Strom BL, Rosenberg L: Use of nonsteroidal antiinflammatory drugs and risk of breast cancer: the Case-Control Surveillance Study revisited. Am J Epidemiol 2005, 162:165-170.

32. Moorman P, Grubber J, Millikan R, Newman B: Association between non-steroidal anti-inflammatory drugs (NSAIDs) and invasive breast cancer and carcinoma in situ of the breast. Cancer Causes Control 2003, 14:915-922.

33. Cotterchio M, Kreiger N, Sloan M, Steingart A: Nonsteroidal antiinflammatory drug use and breast cancer risk. Cancer Epidemiol Biomarkers Prev 2001, 10:1213-1217.

34. Schatzkin A, Subar AF, Thompson FE, Harlan LC, Tangrea J, Hollenbeck AR, Hurwitz PE, Coyle L, Schussler N, Michaud DS, Freedman LS, Brown CC, Midthune D, Kipnis V: Design and serendipity in establishing a large cohort with wide dietary intake distributions: the National Institutes of Health-American Association of Retired Persons Diet and Health Study. Am J Epidemiol 2001, 154:1119-1125.

35. Chandrasekharan NV, Dai $\mathrm{H}$, Roos KLT, Evanson NK, Tomsik J, Elton TS, Simmons DL: COX-3, a cyclooxygenase-1 variant inhibited by acetaminophen and other analgesic/antipyretic drugs: cloning, structure, and expression. Proc Natl Acad Sci USA 2002, 99:13926-13931.

36. ICD-0-3 Coding Materials [http://seer.cancer.gov/icd-o-3/]

37. Michaud DS, Midthune D, Hermansen S, Leitzmann M, Harlan LC, Kipnis V, Schatzkin A: Comparison of cancer registry case ascertainment with SEER estimates and self-reporting in a subset of the NIH-AARP Diet and Health Study. J Registry Manage 2005, 32:70-75.

38. Thiébaut AC, Bénichou J: Choice of time-scale in Cox's model analysis of epidemiologic cohort data: a simulation study. Stat Med 2004, 23:3803-3820.

39. Gail $\mathrm{MH}$, Lubin JH, Rubinstein LV: Likelihood calculations for matched case-control studies and survival studies with tied death times. Biometrika 1981, 68:703-707.

40. Coogan PF, Rao SR, Rosenberg L, Palmer JR, Strom BL, Zauber $A G$, Stolley PD, Shapiro S: The relationship of nonsteroidal anti-inflammatory drug use to the risk of breast cancer. Prev Med 1999, 29:72-76.

41. Harris RE, Namboodiri K, Stellman SD, Wynder EL: Breast cancer and NSAID use: heterogeneity of effect in a case-control study. Prev Med 1995, 24:119-120.

42. Harris RE, Namboodiri KK, Farrar WB: Nonsteroidal antiinflammatory drugs and breast cancer. Epidemiology 1996, 7:203-205.

43. Sharpe CR, Collet JP, McNutt M, Belzile E, Boivin JF, Hanley JA: Nested case-control study of the effects of non-steroidal antiinflammatory drugs on breast cancer risk and stage. $\mathrm{Br} J$ Cancer 2000, 83:112-120.

44. Swede H, Mirand AL, Menezes RJ, Moysich KB: Association of regular aspirin use and breast cancer risk. Oncology 2005, 68:40-47.

45. Curhan GC, Bullock AJ, Hankinson SE, Willett WC, Speizer FE, Stampfer MJ: Frequency of use of acetaminophen, nonsteroidal anti-inflammatory drugs, and aspirin in US women. Pharmacoepidemiol Drug Saf 2002, 11:687-693.

46. Paulose-Ram R, Hirsch R, Dillon C, Losonczy K, Cooper M, Ostchega $Y$ : Prescription and non-prescription analgesic use among the US adult population: results from the third National Health and Nutrition Examination Survey (NHANES III). Pharmacoepidemiol Drug Saf 2003, 12:315-326.

47. Leo C, Faber S, Hentschel B, Höckel M, Horn LC: The status of cyclooxygenase-2 expression in ductal carcinoma in situ lesions and invasive breast cancer correlates to cyclooxygenase-2 expression in normal breast tissue. Ann Diagn Pathol 2006, 10:327-332.

48. Howe LR: Inflammation and breast cancer. Cyclooxygenase/ prostaglandin signaling and breast cancer. Breast Cancer Res 2007, 9:210.

49. Mayne ST, Lippman SM: Cigarettes: a smoking gun in cancer chemoprevention. J Natl Cancer Inst 2005, 97:1319-1321. 